\title{
Article \\ Disruptive NADSYN1 Variants Implicated in Congenital Vertebral Malformations
}

\author{
Jiachen Lin ${ }^{1,2,3,4,+}$, Lina Zhao ${ }^{3,4,5,+}$, Sen Zhao ${ }^{1,2,3,4}$, Shengjie Li ${ }^{3,5}$, Zhengye Zhao ${ }^{1,2,3,4}$, Zefu Chen ${ }^{1,2,3,4}$, \\ Zhifa Zheng $2,3,4,5$, Jiashen Shao ${ }^{1,2,3,4}$, Yuchen Niu ${ }^{3,5}$, Xiaoxin Li ${ }^{3,5}$, Jianguo Terry Zhang ${ }^{1,2,4,5}$ (D), \\ Zhihong $W u^{2,3,4,5}$ and Nan $W u^{1,2,4,5, * 1 D}$
}

check for

updates

Citation: Lin, J.; Zhao, L.; Zhao, S.; Li, S.; Zhao, Z.; Chen, Z.; Zheng, Z.; Shao, J.; Niu, Y.; Li, X.; et al. Disruptive NADSYN1 Variants Implicated in Congenital Vertebral Malformations. Genes 2021, 12, 1615. https://doi.org/10.3390/ genes12101615

Academic Editors: A. J. Agopian and Jennifer E. Posey

Received: 24 August 2021

Accepted: 10 October 2021

Published: 14 October 2021

Publisher's Note: MDPI stays neutral with regard to jurisdictional claims in published maps and institutional affiliations.

Copyright: (c) 2021 by the authors. Licensee MDPI, Basel, Switzerland. This article is an open access article distributed under the terms and conditions of the Creative Commons Attribution (CC BY) license (https:/ / creativecommons.org/licenses/by/ $4.0 /)$.
1 Department of Orthopedic Surgery, Peking Union Medical College Hospital, Peking Union Medical College and Chinese Academy of Medical Sciences, Beijing 100730, China; linjiachen.med@foxmail.com (J.L.); zhaosen830@163.com (S.Z.); zhaozhengye97@163.com (Z.Z.); paragonjeff@163.com (Z.C.); lancetshao@163.com (J.S.); jgzhang_pumch@yahoo.com (J.T.Z.)

2 Beijing Key Laboratory for Genetic Research of Skeletal Deformity, Beijing 100730, China; chivasmo@163.com (Z.Z.); wuzh3000@163.com (Z.W.)

3 Medical Research Center, Peking Union Medical College Hospital, Peking Union Medical College and Chinese Academy of Medical Sciences, Beijing 100730, China; zhaolina19921125@163.com (L.Z.); 1sjlab@163.com (S.L.); nhtniuyuchen@126.com (Y.N.); ldx217@yeah.net (X.L.)

4 Key Laboratory of Big Data for Spinal Deformities, Chinese Academy of Medical Sciences, Beijing 100730, China

5 State Key Laboratory of Complex Severe and Rare Diseases, Peking Union Medical College Hospital, Peking Union Medical College and Chinese Academy of Medical Sciences, Beijing 100730, China

* Correspondence: dr.wunan@pumch.cn

+ These authors contributed equally to this study.

Abstract: Genetic perturbations in nicotinamide adenine dinucleotide de novo (NAD) synthesis pathway predispose individuals to congenital birth defects. The NADSYN1 encodes the final enzyme in the de novo NAD synthesis pathway and, therefore, plays an important role in NAD metabolism and organ embryogenesis. Biallelic mutations in the NADSYN1 gene have been reported to be causative of congenital organ defects known as VCRL syndrome (Vertebral-Cardiac-Renal-Limb syndrome). Here, we analyzed the genetic variants in NADSYN1 in an exome-sequenced cohort consisting of patients with congenital vertebral malformations (CVMs). A total number of eight variants in NADSYN1, including two truncating variants and six missense variants, were identified in nine unrelated patients. All enrolled patients presented multiple organ defects, with the involvement of either the heart, kidney, limbs, or liver, as well as intraspinal deformities. An in vitro assay using COS-7 cells demonstrated either significantly reduced protein levels or disrupted enzymatic activity of the identified variants. Our findings demonstrated that functional variants in NADSYN1 were involved in the complex genetic etiology of CVMs and provided further evidence for the causative NADSYN1 variants in congenital NAD Deficiency Disorder.

Keywords: congenital vertebral malformation; exome sequencing; NADSYN1 gene; congenital NAD Deficiency Disorder

\section{Introduction}

Congenital vertebral malformation (CVM) refers to abnormal development of the spine structure presenting as congenital scoliosis, kyphosis, or other congenital vertebral defects and may occur simultaneously with other birth defects or as part of an underlying genetic syndrome [1,2]. In human embryogenesis, the vertebral column develops at 4-6 weeks of gestation from the paraxial mesoderm (PSM) and is closely related to the spinal cord and other organs originating from mesoderm [3-5]. Multiple organ defects, most frequently involving the cardiac system, urogenital system, limbs, and spinal cord, have a higher occurrence in CVM than in the general population [3]. A genetic component to CVM risk is suspected. However, only 10 20\% of the cases are genetically resolved [6-10]. 
Nicotinamide adenine dinucleotide (NAD) is biologically essential as a key coenzyme in redox reactions participating in cell metabolism, proliferation and inflammation, as well as circadian rhythm [11,12]. In mammals, NAD is de novo synthesized from L-tryptophan or recycled via the salvage synthesis pathway from endogenous NAD metabolites [12]. Recently, genetic perturbations in the de novo NAD synthesis pathway were identified in individuals with birth defects in vertebral, cardiac, renal organs and limbs, namely the VCRL syndrome [MIM: 617660, 617661, 618845] [13,14]. Truncating variants and disruptive missense variants in the genes encoding key enzymes of the de novo synthesis pathway, including $H A A O, K Y N U$ and NADSYN1 were identified, respectively. Further investigation revealed that the genetic deletion of the Haao or Kynu gene, together with deficient dietary NAD precursors during pregnancy, causes VCRL malformations and miscarriages in mice [15]. NADSYN1, encoding the final enzyme in the de novo synthesis pathway, NAD synthetase 1, was reported to be a causative gene for congenital NAD Deficiency Disorder [13]. In a previous study, biallelic variants in NADSYN1 were identified in five individuals from four unrelated families. However, the mutational spectrum of NADSYN1associated congenital disorders has not yet been investigated in a large population cohort.

Here we analyzed the genetic variants in NADSYN1 in an exome-sequenced cohort consisting of Chinese patients diagnosed with CVM and other congenital organ defects. We further performed in vitro functional assays to investigate the effects of these variants on protein expression and enzyme activity. These findings identified the involvement of functional NADSYN1 variants in the complex genetic etiology of CVMs.

\section{Materials and Methods}

\subsection{Patient Recruitment and Clinical Evaluation}

A total of 424 probands diagnosed with CVMs were consecutively enrolled and collected in the cohort between 2009 and 2018 at the Department of Orthopedic Surgery of Peking Union Medical College Hospital, as a part of the Deciphering disorders Involving Scoliosis and COmorbidities (DISCO) study (http:/ / www.discostudy.org/ (accessed on 10 January 2019)). Detailed phenotypic data was recorded. X-ray, computed tomography (CT), and magnetic resonance imaging (MRI) were also performed. Deformities of limbs, spine and spinal cord of relevant cases were evaluated via X-ray plain films by two independent surgeons. The cardiac anomalies were evaluated via ultrasonic cardiography. Urogenital and gastrointestinal anomalies were evaluated via ultrasonography of the abdomen. Patients diagnosed with clinical features of VACTERL association (vertebral defects, anal atresia, cardiac defects, tracheo-esophageal fistula, renal anomalies, and limb abnormalities), namely, the anal atresia and tracheo-esophageal fistula were evaluated and ruled out from the study. Physical examination was performed to evaluate the conditions of each patient's parents. The ethical committee at PUMCH approved the study (IRB number: JS-908). Informed consent was obtained from each participant or their guardians.

\subsection{Exome Sequencing and Variant Interpretation}

Exome sequencing and bioinformatic analysis were conducted. DNA samples were not available for parents. Variants were called, annotated and filtered using the PUMCH developed pipeline (PUMP) as described previously [6]. Rare variants (MAF < 0.001) were selected for analysis based on 1000 Genomes (October 2013), the Exome Aggregation Consortium (ExAC; http:/ / exac.broadinstitute.org (accessed on 10 January 2019)), and the Genome Aggregation Database (gnomAD, http:/ /gnomad.broadinstitute.org/ (accessed on 10 January 2019)). In silico prediction tools, including Sorting Intolerant from Tolerant (SIFT) [16], Polymorphism Phenotyping v2 (Polyphen-2) [17], Genomic Evolutionary Rate Profiling (GERP++) [18] and Combined Annotation Dependent Depletion (CADD) [19] were utilized to predict the deleterious properties of variants. The RefSeq accession numbers of the transcript and corresponding protein isoform of NADSYN1 we used for mutation nomenclature are NM_018161.5 and NP_060631.2, respectively. 


\subsection{Site-Directed Mutagenesis Plasmid Construction}

C-terminal Myc-His-tagged NADSYN1 cDNA (NM_018161.5) in pcDNA3.1+ (Hitrobio Biotechnology, Beijing, China) was generated and used as the template for site-directed mutagenesis following the manufacturer's instructions for the KOD-NEO-PLUS Kit (TOYOBO, Tokyo, Japan). Primers for site-directed mutagenesis at each mutation site were listed in Supplementary Table S1. The mutant plasmids were sequenced on both strands to validate the nucleotide mutation.

\subsection{Plasmid Transfection and Western Blots}

COS-7 cells were acquired from the Institute of Basic Medical Sciences, Chinese Academy of Medical Sciences (Beijing, China). COS-7 cells were cultured in DMEM high glucose (Gibco, Thermo Fisher Scientific, Hudson, NH, USA) supplemented with $10 \%$ fetal bovine serum (Gibco, Thermo Fisher Scientific, Hudson, NH, USA) and penicillinstreptomycin solution (50 U/mL, Gibco, USA). COS-7 cells were grown to $60-70 \%$ confluency in 6-well plates and then transfected with $2 \mu \mathrm{g}$ of pcDNA3.1 NADSYN1 WT and all mutant plasmids using Lipofectamine 3000 reagent (Thermo Fisher Scientific, Vilnius, Lithuania). Eight hours after transfection, DMEM high glucose with 20\% FBS was added into the transfection mix equivalently. Forty-eight hours post-transfection, after washing with cold PBS, the COS-7 cells were lysed with $80 \mu \mathrm{L}$ RIPA buffer (Thermo Fisher Scientific, Hudson, NH, USA) containing protease inhibitor cocktail (Roche, Mannheim, Germany).

Then SDS-PAGE and Western blot analysis were conducted on whole cell lysates by standard methods. A rabbit anti-His-tag antibody (1:1000, AE068, ABclonal, Wuhan, China) was used as the primary antibody and goat anti-rabbit horseradish peroxidase-conjugated secondary antibody (ab6721, Abcam, Cambridge, MA, USA) was applied to detect WT and mutant protein expression levels. Then, the cell lysates were re-probed with horseradish peroxidase-conjugated anti-GAPDH antibody (ab9482, Abcam, Cambridge, MA, USA). NADSYN1 and GAPDH expression level were visualized by automatic chemiluminescence imaging system (C300, Azure Biosystems, Dublin, CA, USA). Then quantification of band intensity was performed by ImageJ (National Institutes of Health, Bethesda, MD, USA). Overall expression levels of WT and mutant NADSYN1 were normalized to GAPDH levels, respectively. Statistical comparisons were conducted with the GraphPad Prism software using the one way ANOVA. Each experiment was repeated three times. $p$-Values $\left.<0.05{ }^{*}\right)$ were considered statistically significant.

\subsection{Expression and Purification of NADSYN1 Protein in Mammalian COS-7 cells}

The COS-7 cells were grown to $60-70 \%$ confluency on $2 \times 15 \mathrm{~cm}$ dishes. Forty-eight hours post-transfection, the cells were lysed on ice for $30 \mathrm{~min}$ with PBS $+1 \%$ TridonX-100 + $1 \% \mathrm{NP}-40+1 \% \mathrm{PMSF}$ (DMSO free), $\mathrm{pH}=7.5$, then freeze-thawed 5 times, and ultrasonicated for 10 cycles. WT and mutant NADSYN1 protein was purified using HisPur ${ }^{\mathrm{TM}}$ Ni-NTA Purification Kit following the manufacturer's instructions (88227, Thermo Fisher Scientific, Hudson, NH, USA). The equilibration, wash, and elution buffers were $10 \mathrm{mM}, 25 \mathrm{mM}$, and $500 \mathrm{mM}$ imidazole respectively. The resin beds were incubated with protein extract for $30 \mathrm{~min}$ on an end-overend rotor at $4{ }^{\circ} \mathrm{C}$. The eluted proteins were quantified by BCA protein assay kit (Sigma-Aldrich, Darmstadt, Germany).

\subsection{Enzymatic Assays of NADSYN1 Protein}

NADSYN1 protein acts as the final enzyme in $\mathrm{NAD}^{+}$biosynthesis, therefore the content of $\mathrm{NAD}^{+}$was measured using an enzymatic assay described in previous studies [13]. The reaction buffer was prepared by mixing $2 \mathrm{mM}$ ATP, $0.2 \mathrm{mg} / \mathrm{mL}$ bovine serum albumin, $5 \mathrm{mM} \mathrm{MgCl}_{2}, 56 \mathrm{mM} \mathrm{KCl}, 50 \mathrm{mM}$ Tris- $\mathrm{HCl}$ (pH 8.0), $20 \mathrm{mM}$ glutamine and $1 \mathrm{mM} \mathrm{NaAD}$. Each reaction system contained $20 \mu \mathrm{L}$ of reaction mix and $0.2 \mu \mathrm{g}$ of protein and was then incubated for $60 \mathrm{~min}$ at $37^{\circ} \mathrm{C}$ and terminated at $95^{\circ} \mathrm{C}$ for $5 \mathrm{~min}$. After centrifugation at $13,000 \mathrm{rpm}$ for $15 \mathrm{~min}$, the supernatants were collected for NAD detection. NAD assays were performed in $900 \mu \mathrm{L}$ of $0.1 \%$ ethanol, $10 \mathrm{mM}$ sodium pyrophosphate and 20 units 
of alcohol dehydrogenase (74931, Sigma-Aldrich, Darmstadt, Germany). The absorbance at $340 \mathrm{~nm}$ was measured before and after a 30-min room temperature using Multiskan FC Microplate Photometer (Thermo Fisher Scientific, Hudson, NH, USA). Standard NAD (0-100 nmol) was measured under the same conditions. Statistical analysis of NADSYN1 enzymatic activity were conducted in GraphPad Prism software using one way ANOVA method. Each experiment was repeated three times. $p$-Values $<0.05\left(^{*}\right)$ were considered statistically significant.

\section{Results}

3.1. Identification and Prioritization of NADSYN1 Variants Implicated in DISCO Cohort

Exome sequencing (ES) was performed on 424 sporadic CVM cases from the DISCO study. We identified a total of eight rare NADSYN1 heterozygous nonsynonymous variants in nine unrelated patients (Table 1). These variants were classified clinically as having unknown functional consequences and, therefore, as VUS in accordance with the American College of Medical Genetics and Genomics (ACMG) guidelines for classification of variant pathogenicity [20]. No pathogenic variants of CVM were identified previously in all of the enrolled patients.

Six missense and two truncating variants were found in 9 unrelated patients. Two of these unrelated patients (SCO2003P0106 and SCO2003P0213) carried the same missense variant (Table 1). Among the six missense variants, three of them were predicted to be deleterious by both SIFT prediction and Polyphen-2 software, with the CADDPHRED score >20 (c.1037G > A, p.Arg346Gln; c.1762G > A, p.Glu588Lys; c.709G > A, p.Gly237Arg, with the CADD score of 29.6, 20.6, and 23.4, respectively, Table 2). Moreover, the GERP rejected substitution scores of the three variants are $>4$, suggesting the variants might be functionally disruptive (5.11,4.83, and 4.64, respectively). Another variant, c.1511G > A(p.Arg504Gln) was also predicted to be deleterious, with a CADD score of 21.1 and a GERP++ score of 3.98. As for the other two missense mutations, c.232G > A(p. Val78Ile) and c.2083G > A(p. Glu695Lys), the in silico prediction was benign/tolerated, with CADD scores of 11.48 and 18.37 and GERP++ scores of 2.99 and 4.35 , respectively (Table 2 ).

\subsection{Phenotypic Characteristics of Individuals with NADSYN1 Variants}

The clinical features of the enrolled individuals are summarized in Table 1. The parents of the enrolled individuals were phenotypically normal based on the physical examination. Vertebral malformations included hemivertebrae, and wedge-shaped vertebrae at the lumbar and thoracic level. Patient SCO2003P0286 presented with preaxial polydactyly. Notably, all of the enrolled patients had extraskeletal abnormalities. Three patients were diagnosed with heart defects, including mitral insufficiency (SCO2003P1980), bicuspid aortic valve (SCO2003P0240), or mitral valve prolapse (SCO2003P0106). Two patients presented renal aplasia (SCO2003P0164 and SCO2003P0286). Four patients were found to have diastematomyelia (SCO2003P0298, SCO2003P1273, SCO2003P0106 and SCO2003P0213), while tethered cords were found among SCO2003P0096 and SCO2003P0213. Additionally, multiple hepatic polycysts were recorded in two of the patients, namely, SCO2003P0298 and SCO2003P0096. 


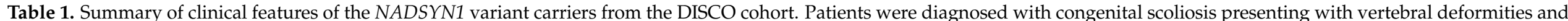

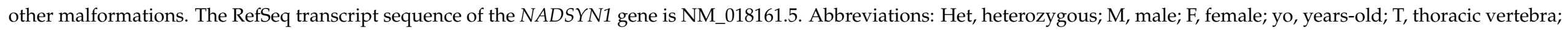
$\mathrm{L}$, lumbar vertebra.

\begin{tabular}{|c|c|c|c|c|c|c|}
\hline Patient Number & Variant & Zygosity & Sex & Age at Admission & Vertebral Deformities & Other Malformations \\
\hline SCO2003P0298 & c.232G > A(p.Val78Ile) & Het & $\mathrm{F}$ & 12 yo & $\begin{array}{l}\mathrm{T} 3, \mathrm{~T} 5, \mathrm{~T} 6, \mathrm{~T} 7, \mathrm{~T} 8 \text { vertebral malfor- } \\
\text { mation and unsegmentation, } \\
\mathrm{T} 10-\mathrm{T} 11 \text { unsegmentation }\end{array}$ & $\begin{array}{c}\text { Diastematomyelia, multiple } \\
\text { hepatic polycysts, } \\
\text { maxillary sinusitis }\end{array}$ \\
\hline SCO2003P1273 & c.709G > A(p.Gly237Arg) & Het & M & 13 yo & $\begin{array}{l}\text { T2-L3 vertebral malformation } \\
\text { and unsegmentation }\end{array}$ & $\begin{array}{l}\text { Diastematomyelia, spina } \\
\text { bifida, mucocele }\end{array}$ \\
\hline SCO2003P0164 & c.861delT(p.Arg288GlufsTer14) & Het & M & 11 yo & $\begin{array}{l}\text { T6 butterfly vertebra, } \\
\text { T7 hemivertebra, T5-T9 fused } \\
\text { vertebral plate; T7 rib absent, } \\
\text { T9-T10 fused rib }\end{array}$ & Renal aplasia (left) \\
\hline SCO2003P0240 & c.1037G > A(p.Arg346Gln) & Het & M & 6 yo & T10, T11, T12 hemivertebrae & Bicuspid aortic valve \\
\hline SCO2003P1980 & c.1216C > T(p.Arg406Ter) & Het & $\mathrm{F}$ & 1 yo & T6-T10 unsegmentation & Mitral insufficiency \\
\hline SCO2003P0096 & c.1511G > A(p.Arg504Gln) & Het & $\mathrm{F}$ & 9 yo & $\begin{array}{l}\text { L2 wedge-shape vertebra, } \\
\text { L3 wedge-shape vertebra, } \\
\text { L1-L4 unsegmentation }\end{array}$ & $\begin{array}{l}\text { Tethered cord, multiple hepatic } \\
\text { polycysts, splenomegaly }\end{array}$ \\
\hline SCO2003P0286 & c.1762G > A(p.Glu588Lys) & Het & $\mathrm{F}$ & 1 yo & $\begin{array}{l}\text { T3-T8 vertebral malformation } \\
\text { and unsegmentation }\end{array}$ & $\begin{array}{l}\text { Renal aplasia (left), polydactyly } \\
\text { (left), dilatation of central canal of } \\
\text { spinal cord }\end{array}$ \\
\hline SCO2003P0106 & c.2083G > A(p.Glu695Lys) & Het & M & 10 yo & $\begin{array}{l}\text { T7, T8, T9 butterfly vertebrae, } \\
\text { T6-T10 unsegmentation }\end{array}$ & $\begin{array}{l}\text { Diastematomyelia, mitral } \\
\text { valve prolapse }\end{array}$ \\
\hline SCO2003P0213 & c.2083G > A(p.Glu695Lys) & Het & M & 22 yo & $\begin{array}{l}\text { T8-T9 unsegmentation, } \\
\text { T9-T10 rib bifurcation }\end{array}$ & Diastematomyelia, tethered cord \\
\hline
\end{tabular}


Table 2. Sequence variants in NADSYN1 and their statistics in gnomAD statistics, in silico prediction scores, and in vitro assay results. The RefSeq transcript sequence of the NADSYN1 gene is NM_018161.5. Abbreviations: NA, not available.

\begin{tabular}{|c|c|c|c|c|c|c|c|}
\hline $\begin{array}{l}\text { Patient } \\
\text { Number }\end{array}$ & $\begin{array}{l}\text { cDNA } \\
\text { Change }\end{array}$ & $\begin{array}{l}\text { Protein } \\
\text { Change }\end{array}$ & $\begin{array}{l}\text { gnomAD * } \\
\text { Allele } \\
\text { Frequency }\end{array}$ & $\begin{array}{c}\text { SIFT } \\
\text { Prediction } \\
\text { Score }\end{array}$ & Polyphen-2 & GERP++ & $\begin{array}{c}\text { CADD } \\
\text { PHRED } \\
\text { Score }\end{array}$ \\
\hline SCO2003P0298 & $232 \mathrm{G}>\mathrm{A}$ & Val78Ile & 0 & 0.5 & 0.013 & 4.35 & 18.37 \\
\hline SCO2003P1273 & $709 \mathrm{G}>\mathrm{A}$ & Gly237Arg & 0 & 0 & 0.974 & 4.64 & 23.4 \\
\hline SCO2003P0164 & 861del & Arg288GlufsTer14 & 0 & NA & NA & NA & NA \\
\hline SCO2003P0240 & $1037 \mathrm{G}>\mathrm{A}$ & Arg346Gln & 0 & 0.01 & 0.994 & 5.11 & 29.6 \\
\hline SCO2003P1980 & $1216 \mathrm{C}>\mathrm{T}$ & Arg406Ter & 0.0000083 & NA & NA & 3.05 & 42 \\
\hline SCO2003P0096 & $1511 G>A$ & Arg504Gln & 0.00013 & 0.06 & 0.378 & 3.98 & 21.1 \\
\hline SCO2003P0286 & $1762 \mathrm{G}>\mathrm{A}$ & Glu588Lys & 0 & 0 & 1 & 4.83 & 20.6 \\
\hline $\begin{array}{l}\text { SCO2003P0106/ } \\
\text { SCO2003P0213 }\end{array}$ & $2083 G>A$ & Glu695Lys & 0.00022 & 0.21 & 0.01 & 2.99 & 11.48 \\
\hline
\end{tabular}

* Dataset based on highest MAF in population.

\subsection{Distribution of the NADSYN1 Variants on the Protein}

A protein domain diagram of NADSYN1 (Figure 1A) highlights the positions of the identified variants. As demonstrated, the frameshift indel at Arg288 is predicted to cause complete loss of the NAD synthetase domain in the translated protein. Similarly, the stop-gain mutation at Arg406 is predicted to induce abruption of NAD synthetase encoding. The four predictively deleterious variants at Gly237, Arg346, Gln504 and Glu588, highlighted in red, are located in an evolutionarily conserved sequence across species (Figure 1C). Notably, the variant p.Glu588Lys presents within the conserved P2 loop of NAD synthetase, similar to the previously reported functional variant p.Ala573Thr in VCRL patients [13]. Additionally, p.Arg504Gln is also located next to the P2 loop site. In contrast, the variant p.Arg346Gln surrounds the ATP-binding site at amino acid Val360 [21]. We then visualized the space distribution of variants in the 3D model using the SwissModel and PyMOL (PDB ID: 6OFB, https: / / pymol.org (accessed on 4 January 2021)) [22] (Figure 1B). The variant p.Gly237Arg, as demonstrated in the glutaminase domain, is found to be close to the ATP-binding domain the same as variant p.Arg346Gln. These findings suggested that the above missense variants located closely to the critical functional domains of NADSYN1 protein.

\subsection{Functional Assessment of the NADSYN1 Variants}

To test the effect of all the NADSYN1 variants on protein function, we overexpressed either human NADSYN1 wild-type or mutant protein in COS-7 cells. Forty-eight hours after transfection, total cell lysate was collected and plasma protein extracted. We then performed SDS-PAGE and Western blot analysis on the whole cell lysate from WT and the respective mutants (Figure 2A,C). The results demonstrated a low expression level of endogenous NADSYN1 protein in COS-7 cell line. A loss of expression of complete protein after transfecting the truncating variants, c.861delT(p.Arg288GlufsTer14) and c.1216C > $\mathrm{T}$ (p.Arg406Ter) was noted (Figure 2A, both $p<0.001$ ), possibly due to the non-sense mediated decay (NMD) effects in the cells. Compared to wild-type, significantly decreased expression of variant c.232G > A(p.Val78Ile) $(p<0.05)$, c.1037G > A (p.Arg346Gln) $(p<0.001)$, and c.2083G $>$ A(p.Glu695Lys) $(p<0.05)$ were observed, suggesting a potentially decreased protein stability caused by the variants. The other three variants, c.709G > A(p.Gly237Arg), c.1511G > A(p.Arg504Gln), and c.1762G > A(p.Glu588Lys) did not show altered protein expression levels as compared to that of the wild type. 
A
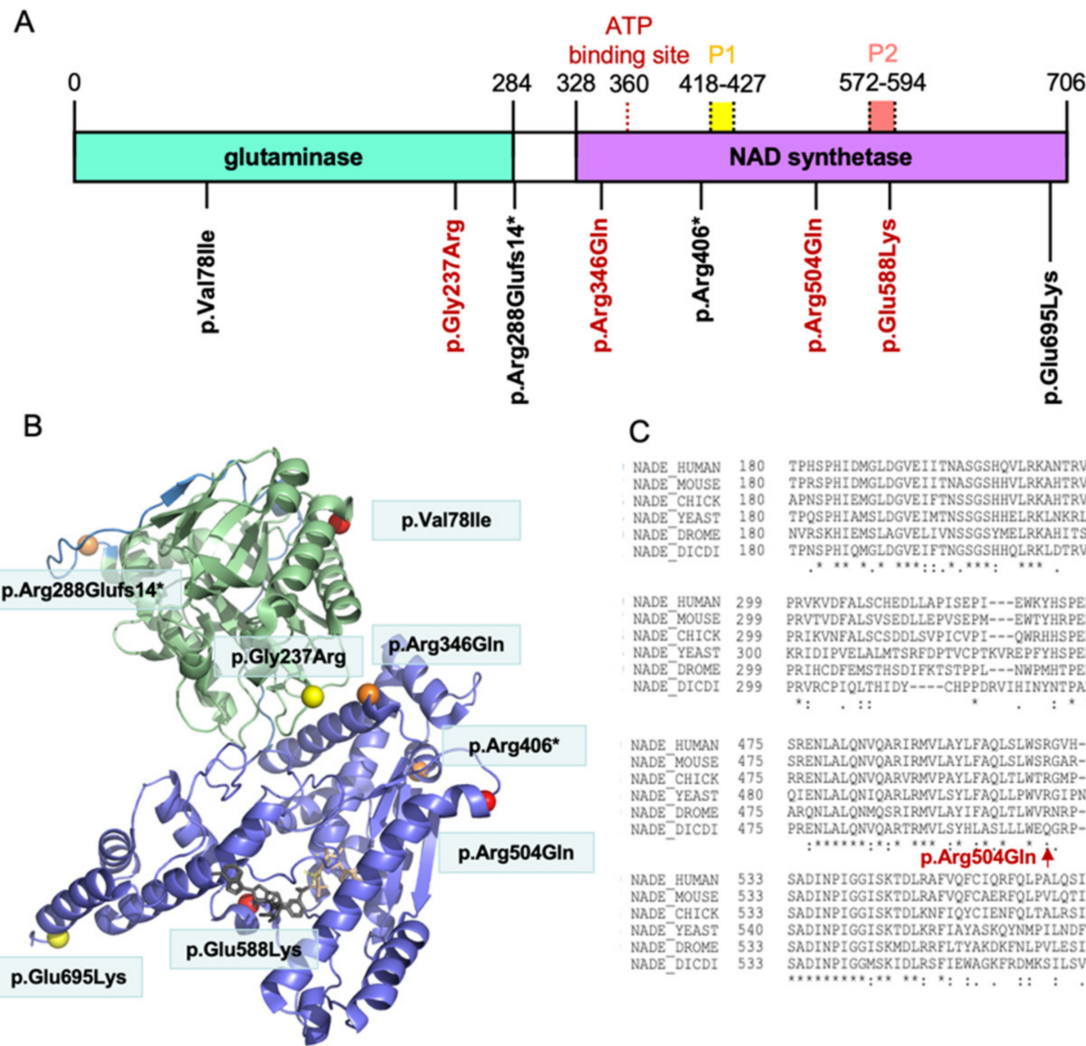

C

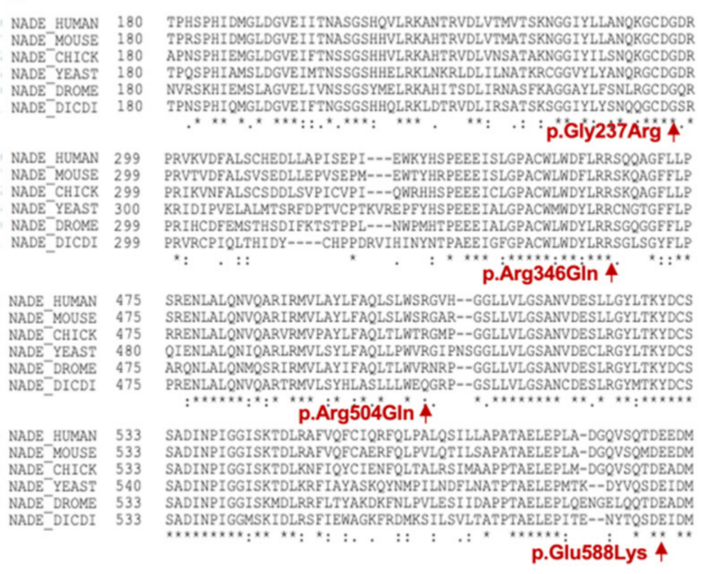

Figure 1. (A) Protein domain diagram of human NADSYN1 and the position of identified variants. The glutaminase domain is labeled in green, NAD synthetase domain in purple, P1 loop in yellow, P2 loop in pink, and ATP binding site in red, respectively. The RefSeq transcript sequence of NADSYN1 gene is NM_018161.5; PDB accession code: 6OFB. (B) Space distribution of variants in 3D model using Swiss-Model and PyMOL (https: / pymol.org (accessed on 4 January 2021)). (C) Position of the variants (red) relative to an evolutionarily conserved region using ClustalW sequence alignment (https:/ / www.genome.jp/tools-bin/clustalw (accessed on 8 April 2021)).

Previous studies have demonstrated that deleterious NADSYN1 variants could generate NAD synthetase with impaired enzymatic activity [13]. We further tested the enzymatic activities of wild-type and mutants using a previously described method [13]. We were not able to generate enough purified NADSYN1 from the variant c.1037G > A(p.Arg346Gln) due to the low protein expression. The enzymatic activities of NAD of wild-type and five other mutants were assessed (Figure 2D). We found a significantly decreased level of mutant enzymatic activity. The mutant c.232G > A(p.Val78Ile) and c.1762G > A(p.Glu588Lys) demonstrated a 100-150 fold decrease compared to wild-type (both $p<0.001$ ), and the c.1511G > A(p.Arg504Gln) demonstrated an 20-fold decrease in enzymatic activities ( $p<0.001)$. Additionally, c.2083G > A(p.Glu695Lys) demonstrated an 3.75-fold and c.709G $>\mathrm{A}$ (p.Gly237Arg) an 1.5-fold decrease in enzymatic activity (both $p<0.001$ ). Notably, both variants on the glutaminase domain (c.232G > A, c.709G > A) and NAD synthetase domain (c.1511G > A, c.1762G > A and c.2083G > A) (Figure 1) resulted in a significantly decreased NAD synthesis ability. 
A

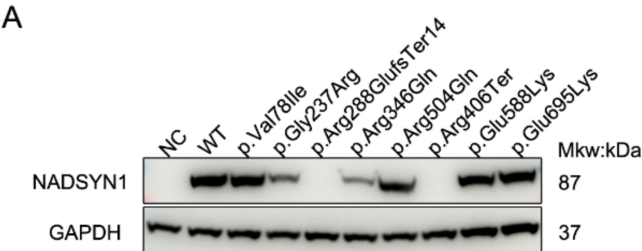

C

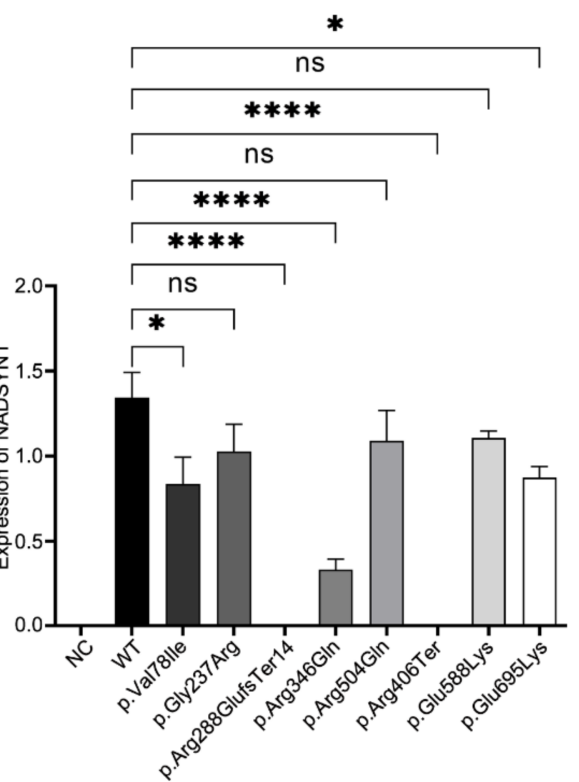

B

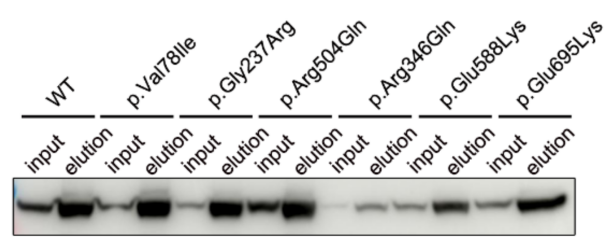

D

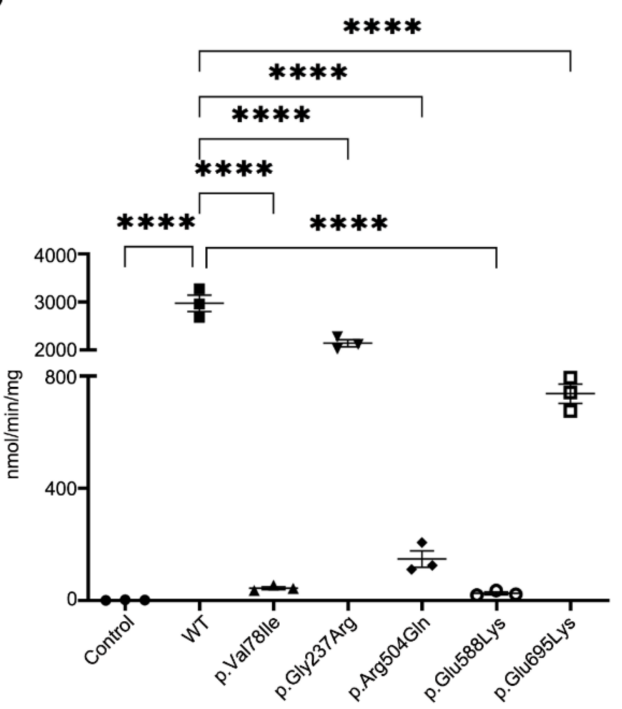

Figure 2. (A) Expression of NADSYN1 protein in cells transfected with wild type (WT) and mutant NADSYN1 plasmid. (B) The western blot shows the content of the NADSYN1 protein before and after protein purification. (C)Quantification of the NADSYN1 WT and mutant protein based on western blot results. Then band intensity was measured by ImageJ based on ImageJ software. (D) Nicotinamide adenine dinucleotide synthetase activity of purified mutant protein compared to wild-type (WT) NADSYN1 protein. Abbreviations: NC, negative control; ns, non-significant. The results are shown as the mean \pm standard error of three independent experiments. $p$-Values $<0.05$ $\left({ }^{*}\right), 0.0001{ }^{(* * * *)}$ were considered significant.

\section{Discussion}

In the present study, we identified eight potentially disruptive variants in a total number of nine CVM patients, with subsequent functional assessment. All patients presented with CVMs and other defects, either in heart, kidney, limbs, or spinal cord. We further evaluated the pathogenicity of these variants using in silico prediction methods, and tested the protein expression level and enzymatic activity in the COS-7 cell line. These findings provided further evidence of the involvement of NAD deficiency in congenital organ defects.

Nicotinamide adenine dinucleotide (NAD) is essential for mammals and serves endogenously as a key coenzyme in redox reactions and a precursor for cell metabolism, and also a substrate for protein modifications [11,12]. Congenital NAD deficiency due to genetic perturbations and dietary deficiency of NAD precursors would impair organogenesis both in humans and mice [14,15]. It was reported that genetic loss of non-redundant de novo NAD synthesis genes, KYNU and HAAO, was associated with the VCRL syndrome, which is characterized by congenital vertebral malformations and other organ defects. The vertebral phenotype of humans was reproduced in mouse models with genetic deletion of Kynu and Haao, complicated with dietary deficiency of NAD precursors in pregnant mice. These data suggested that congenital NAD Deficiency Disorder may be caused by a gene-environment interaction. 
NADSYN1 encodes the final enzyme of the NAD de novo synthesis pathway [23]. The role of NADSYN1 during organ development has not yet been elucidated. Notably, all five individuals presented in a previous study did not survive more than three months postnatally [13]. NADSYN1, like many non-redundant genes in the NAD synthesis pathway, appears tolerant to heterozygous missense mutations in humans. Here, we present nine individuals carrying heterozygous deleterious variants of NADSYN1 with multiple organ defects who survived postnatally with relatively good quality of life (admitted to the hospital from age 1 to 22 years). In addition to CVM, all patients in this study presented with other organ defects involving either the heart, limbs, kidney, liver or spinal cord. Notably, two of the patients presented with multiple hepatic polycysts, which has not been previously reported in NAD Deficiency Disorder cases. Considering that congenital NAD deficiency disorder is caused by a gene-environment interaction, we propose that functional NADSYN1 variants are involved in CVMs with variable penetrance and expressivity.

The functional NADSYN1 protein contains glutaminase and synthetase domains [21] and catalyzes the ATP-dependent formation of NAD+ from nicotinic acid adenine dinucleotide $(\mathrm{NaAD}+)$ at the synthetase domain using the ammonia generated from the glutaminase domain $[23,24]$. It has been previously reported that variants in both the glutaminase (p. Cys49Arg) domain and synthetase domain (p. Ala573Thr) will cause a significant decrease in NAD synthesis activities of encoded NADSYN1 [13]. To validate the proposed hypothesis, we performed in vitro assays to evaluate the consequences of NADSYN1 variants located in different domains. One frameshift variant, c.861delT (p. Arg288GlufsTer14), led to complete loss of the NAD synthetase domain in the translated protein (Figure 2A,B). Another possible explanation is that the non-sense mediated decay (NMD) is activated in COS-7 cells to mediate the degeneration of mutant mRNA transcripts from variant c.861delT(p. Arg288GlufsTer14) and c.1216C > T(p.Arg406Ter). Two missense variants in the NAD synthetase domain, c.1511G > A(p. Arg504Gln) and c.1762G > A(p. Glu588Lys), are structurally close to the P2 loop, the active site for NAD synthetase [21], significantly decreasing the enzymatic activity (Figure 1A). Consistent with previous results, the missense variant in the glutaminase domain, c.232G > A(p. Val78Ile), demonstrated significantly decreased enzymatic activity [13]. However, given the fact that congenital Deficiency Disorder is known to be caused by biallelic variants in NADSYN1, we still consider these variants as uncertain significance.

As a multifactorial disease with a prevalence of $\sim 1 / 1000$ live births $[1,7]$, genetic perturbations, gene-environment interactions, and epigenetic changes are suspected to play a role in the etiology of CVMs $[4,8]$. Previously, we have reported the mutational burden and potential oligogenic models underpinning CVM development, as well as compound heterozygous inheritance model in TBX6-associated congenital scoliosis (TACS) $[9,25,26]$. The combined effect of deleterious variants in multiple genes might synergistically lead to the development of the malformations, which gives insight into the complex diseasecausing model of CVMs. Apart from genetic perturbations, hypoxia, insufficient folate acid supply, and teratogenic drug use during pregnancy are also considered to be potential CVM risk factors [8]. Considering the complexity of NAD-involved biological processes, the exact mechanism underpinning NAD-mediated organogenesis defects remains to be elucidated. As anticipated, defects in organs originating from the mesoderm in the cardiac system and urogenital system, as well as limbs, often co-occur with somitogenesis defects in other congenital disorders [3,5,27], highlighting the vital role of NADSYN1 during embryogenesis of the mesoderm.

In summary, functional variants in NADSYN1 were involved in the complex genetic etiology of CVMs. Further investigation of congenital NAD Deficiency Disorder will help to understand the pathogenesis of syndromic congenital defects involving CVMs. 
Supplementary Materials: The following are available online at https:/ / www.mdpi.com/article/10 $.3390 /$ genes12101615/s1. The primers for site-directed mutagenesis of the NADSYN1 plasmids are summarized in Supplementary Table S1.

Author Contributions: Conceptualization, J.L., L.Z., J.T.Z., Z.W. and N.W.; methodology, J.L. and L.Z.; software, S.Z.; validation, J.L., L.Z. and S.L.; formal analysis, J.L., J.S., N.W., S.Z. and S.L.; investigation, L.Z., Y.N., X.L. and J.L.; resources, S.Z., J.L., Z.C., Z.Z. (Zhengye Zhao), Z.Z. (Zhifa Zheng) and J.T.Z.; data curation, S.Z., J.S., Y.N. and X.L.; writing—original draft preparation, J.L. and L.Z.; writing-review and editing, J.T.Z., Z.W. and N.W.; visualization, S.L.; supervision, J.T.Z., Z.W. and N.W.; project administration, J.T.Z., Z.W. and N.W.; funding acquisition, J.T.Z., Z.W. and N.W. All authors have read and agreed to the published version of the manuscript.

Funding: This research was funded in part by the Beijing Natural Science Foundation (JQ20032 to N.W., 7191007 to Z.W. and L192015 to J.T.Z.), the National Natural Science Foundation of China (81802121 to S.L., 81822030 and 82072391 to N.W., 81930068 and 81772299 to Z.W. and 81672123 and 81972037 to J.T.Z.), Capital's Funds for Health Improvement and Research (2020-4-40114 to N.W.), the Tsinghua University-Peking Union Medical College Hospital Initiative Scientific Research Program, Nonprofit Central Research Institute Fund of Chinese Academy of Medical Sciences (No. 2019PT320025).

Institutional Review Board Statement: The study was approved by the ethics committee of Peking Union Medical College Hospital (IRB number: JS-908).

Informed Consent Statement: Written consent to use the clinical and genetic data in this report was obtained from the adult patients and from parents/guardians of all children included in this study.

Data Availability Statement: The datasets used and/or analyzed during the current study are available from the corresponding authors upon reasonable request.

Acknowledgments: We thank the Nanjing Geneseeq Technology Inc. for their technical help in sequencing and the Ekitech Technology Inc. for their technical support in database and data management.

Conflicts of Interest: There is no competing interests to declare for all authors.

\section{References}

1. Wynne-Davies, R. Congenital vertebral anomalies: Aetiology and relationship to spina bifida cystica. J. Med. Genet. 1975, 12, 280-288. [CrossRef]

2. Giampietro, P.F.; Dunwoodie, S.L.; Kusumi, K.; Pourquie, O.; Tassy, O.; Offiah, A.C.; Cornier, A.S.; Alman, B.A.; Blank, R.D.; Raggio, C.L.; et al. Progress in the understanding of the genetic etiology of vertebral segmentation disorders in humans. Ann. N. Y. Acad. Sci. 2009, 1151, 38-67. [CrossRef]

3. Furdock, R.; Brouillet, K.; Luhmann, S.J. Organ System Anomalies Associated with Congenital Scoliosis: A Retrospective Study of 305 Patients. J. Pediatr. Orthop. 2019, 39, e190-e194. [CrossRef]

4. Pahys, J.M.; Guille, J.T. What's New in Congenital Scoliosis? J. Pediatr. Orthop. 2018, 38, e172-e179. [CrossRef]

5. Chen, N.; Zhao, S.; Jolly, A.; Wang, L.; Pan, H.; Yuan, J.; Chen, S.; Koch, A.; Ma, C.; Tian, W.; et al. Perturbations of genes essential for Mullerian duct and Wolffian duct development in Mayer-Rokitansky-Kuster-Hauser syndrome. Am. J. Hum. Genet. 2021, 108, 337-345. [CrossRef]

6. Zhao, S.; Zhang, Y.; Chen, W.; Li, W.; Wang, S.; Wang, L.; Zhao, Y.; Lin, M.; Ye, Y.; Lin, J.; et al. Diagnostic yield and clinical impact of exome sequencing in early-onset scoliosis (EOS). J. Med. Genet. 2021, 58, 41-47. [CrossRef]

7. Wu, N.; Ming, X.; Xiao, J.; Wu, Z.; Chen, X.; Shinawi, M.; Shen, Y.; Yu, G.; Liu, J.; Xie, H.; et al. TBX6 null variants and a common hypomorphic allele in congenital scoliosis. N. Engl. J. Med. 2015, 372, 341-350. [CrossRef]

8. Sparrow, D.B.; Chapman, G.; Smith, A.J.; Mattar, M.Z.; Major, J.A.; O’Reilly, V.C.; Saga, Y.; Zackai, E.H.; Dormans, J.P.; Alman, B.A.; et al. A mechanism for gene-environment interaction in the etiology of congenital scoliosis. Cell 2012, 149, 295-306. [CrossRef]

9. Liu, J.; Wu, N.; Deciphering Disorders Involving Scoliosis and COmorbidities (DISCO) Study; Yang, N.; Takeda, K.; Chen, W.; Li, W.; Du, R.; Liu, S.; Zhou, Y.; et al. TBX6-associated congenital scoliosis (TACS) as a clinically distinguishable subtype of congenital scoliosis: Further evidence supporting the compound inheritance and TBX6 gene dosage model. Genet. Med. 2019, 21, 1548-1558. [CrossRef]

10. Chen, W.; Lin, J.; Wang, L.; Li, X.; Zhao, S.; Liu, J.; Akdemir, Z.C.; Zhao, Y.; Du, R.; Ye, Y.; et al. TBX6 missense variants expand the mutational spectrum in a non-Mendelian inheritance disease. Hum. Mutat. 2020, 41, 182-195. [CrossRef]

11. Rajman, L.; Chwalek, K.; Sinclair, D.A. Therapeutic Potential of NAD-Boosting Molecules: The In Vivo Evidence. Cell Metab. 2018, 27, 529-547. [CrossRef] 
12. Nikiforov, A.; Kulikova, V.; Ziegler, M. The human NAD metabolome: Functions, metabolism and compartmentalization. Crit. Rev. Biochem. Mol. Biol. 2015, 50, 284-297. [CrossRef]

13. Szot, J.O.; Campagnolo, C.; Cao, Y.; Iyer, K.R.; Cuny, H.; Drysdale, T.; Flores-Daboub, J.A.; Bi, W.; Westerfield, L.; Liu, P.; et al. Bi-allelic Mutations in NADSYN1 Cause Multiple Organ Defects and Expand the Genotypic Spectrum of Congenital NAD Deficiency Disorders. Am. J. Hum. Genet. 2020, 106, 129-136. [CrossRef]

14. Shi, H.; Enriquez, A.; Rapadas, M.; Martin, E.; Wang, R.; Moreau, J.; Lim, C.K.; Szot, J.O.; Ip, E.; Hughes, J.N.; et al. NAD Deficiency, Congenital Malformations, and Niacin Supplementation. N. Engl. J. Med. 2017, 377, 544-552. [CrossRef]

15. Cuny, H.; Rapadas, M.; Gereis, J.; Martin, E.; Kirk, R.B.; Shi, H.; Dunwoodie, S.L. NAD deficiency due to environmental factors or gene-environment interactions causes congenital malformations and miscarriage in mice. Proc. Natl. Acad. Sci. USA 2020, 117, 3738-3747. [CrossRef]

16. Vaser, R.; Adusumalli, S.; Leng, S.N.; Sikic, M.; Ng, P.C. SIFT missense predictions for genomes. Nat. Protoc. 2016, 11, 1-9. [CrossRef] [PubMed]

17. Adzhubei, I.A.; Schmidt, S.; Peshkin, L.; Ramensky, V.E.; Gerasimova, A.; Bork, P.; Kondrashov, A.S.; Sunyaev, S.R. A method and server for predicting damaging missense mutations. Nat. Methods 2010, 7, 248-249. [CrossRef] [PubMed]

18. Cooper, G.M.; Stone, E.A.; Asimenos, G.; Program, N.C.S.; Green, E.D.; Batzoglou, S.; Sidow, A. Distribution and intensity of constraint in mammalian genomic sequence. Genome Res. 2005, 15, 901-913. [CrossRef]

19. Kircher, M.; Witten, D.M.; Jain, P.; O’Roak, B.J.; Cooper, G.M.; Shendure, J. A general framework for estimating the relative pathogenicity of human genetic variants. Nat. Genet. 2014, 46, 310-315. [CrossRef]

20. Richards, S.; Aziz, N.; Bale, S.; Bick, D.; Das, S.; Gastier-Foster, J.; Grody, W.W.; Hegde, M.; Lyon, E.; Spector, E.; et al. Standards and guidelines for the interpretation of sequence variants: A joint consensus recommendation of the American College of Medical Genetics and Genomics and the Association for Molecular Pathology. Genet. Med. 2015, 17, 405-424. [CrossRef]

21. Chuenchor, W.; Doukov, T.I.; Chang, K.T.; Resto, M.; Yun, C.S.; Gerratana, B. Different ways to transport ammonia in human and Mycobacterium tuberculosis NAD(+) synthetases. Nat. Commun. 2020, 11, 16. [CrossRef] [PubMed]

22. Waterhouse, A.; Bertoni, M.; Bienert, S.; Studer, G.; Tauriello, G.; Gumienny, R.; Heer, F.T.; de Beer, T.A.P.; Rempfer, C.; Bordoli, L.; et al. SWISS-MODEL: Homology modelling of protein structures and complexes. Nucleic Acids Res. 2018, 46, W296-W303. [CrossRef] [PubMed]

23. Hara, N.; Yamada, K.; Terashima, M.; Osago, H.; Shimoyama, M.; Tsuchiya, M. Molecular identification of human glutamine- and ammonia-dependent NAD synthetases. Carbon-nitrogen hydrolase domain confers glutamine dependency. J. Biol. Chem. 2003, 278, 10914-10921. [CrossRef] [PubMed]

24. Massiere, F.; Badet-Denisot, M.A. The mechanism of glutamine-dependent amidotransferases. Cell Mol. Life Sci. 1998, 54, $205-222$. [CrossRef] [PubMed]

25. Yang, Y.; Zhao, S.; Zhang, Y.; Wang, S.; Shao, J.; Liu, B.; Li, Y.; Yan, Z.; Niu, Y.; Li, X.; et al. Mutational burden and potential oligogenic model of TBX6-mediated genes in congenital scoliosis. Mol. Genet. Genom. Med. 2020, 8, e1453. [CrossRef] [PubMed]

26. Li, Z.; Zhao, S.; Cai, S.; Zhang, Y.; Wang, L.; Niu, Y.; Li, X.; Hu, J.; Chen, J.; Wang, S.; et al. The mutational burden and oligogenic inheritance in Klippel-Feil syndrome. BMC Musculoskelet Disord. 2020, 21, 220. [CrossRef]

27. Chen, N.; Pan, H.; Luo, G.; Wang, P.; Xie, Z.; Hua, K.; Luo, X.; Huang, X.; Liu, Q.; Sun, L.; et al. Clinical characteristics of 1055 Chinese patients with Mayer-Rokitansky-Kuster-Hauser syndrome: A nationwide multicentric study. Fertil. Steril. 2021, 116, 558-565. [CrossRef] 